\title{
Professor Andrzej Kossakowski awarded the Polish Nobel Prize
}

DOI: http://dx.doi.org/10.12775/ZN.2019.018

Professor Andrzej Kossakowski was awarded the 2019 Foundation for Polish Science (FNP) Prize in the field of mathematical, physical and engineering sciences for "developing the theory of open quantum systems". He joined the list of distinguished Polish physicists already awarded the FNP Prize.

Andrzej Kossakowski was born in 1938 in Lwów (now Lviv, Ukraine). His scientific career was from the very beginning associated with Nicolaus Copernicus University in Toruń, where between 1955 and 1960 he studied physics, obtained $\mathrm{PhD}$ in theoretical physics in 1966, and became a professor in 1979. Kossakowski's research has always dealt with fundamental problems of quantum physics. To fully appreciate his achievements let us briefly recall the main building blocks of quantum mechanics. Quantum mechanics was born at the beginning of the twentieth century and had a tremendous impact on our understanding of the structure of the world. In particular, quantum mechanics explained atomic spectra of a number of elements observed in laboratories. These spectra were completely unknown to classical physics and an explanation of these phenomena called for a completely new theory. The laws of classical physics work perfectly in macroscale, but they are fundamentally wrong in microscale, i.e. in the world of atoms, molecules and elementary particles. The founders of this new theory had to abandon existing intuitions, habits and even the language used in physics for many decades. In 1926 Erwin Schrödinger proposed a new equation which described the physical properties of atoms and molecules. Schrödinger's equation even enables one to describe the evolution of quantum states represented by wave functions $\psi$ :

$$
i \hbar \dot{\psi}=H \psi,
$$

where $\hbar$ stands for Planck constant, and $H$ is the Hamiltonian - basic object that encodes all properties of the quantum system ( $i$ is an imaginary unit, i.e. $i^{2}=-1$. Interestingly, quantum physics, as opposed to classical physics, needs complex numbers!). Equation (1) is one of the most fundamental equations in physics. Every student of physics studies the properties of this equation in the basic quantum mechanics. In 1927 Lev Landau, the Soviet physicist from Petersburg 
(formerly Leningrad) expanded Schrödinger's equation for mixed quantum states represented by density operators. The corresponding equation is known as von Neumann equation (John von Neumann formalised the Landau observation. We actually proposed the consistent mathematical formulation of the new quantum theory in the 1930s). The von Neumann equation reads as follows

$$
i \hbar \dot{\rho}=[H, \rho]
$$

where $\rho$ now denotes the density operator, one of Kossakowski's favorite objects. In 1928 Paul Dirac (one the greatest physicists ever) finally proposed a relativistic generalisation of Schrödinger's equation (consistent with Einstein's theory of relativity). A substantial contribution was also made by Werner Heisenberg, who in 1925 developed the so-called matrix mechanics (later shown by Dirac to be fully equivalent to the wave mechanics proposed by Schrödinger). Heisenberg proposed an equation describing the time evolution of a physical quantity (called quantum observable) represented by an operator (matrix) $X$

$$
i \hbar \dot{X}=-[H, X]
$$

Heisenberg was awarded the Nobel Prize in physics in 1932, Schrödinger and Dirac in 1933, and Landau in 1962 (actually, Landau was awarded the prize for the development of a mathematical theory of superfluidity).

Equations developed by Schrödinger (1), Landau and von Neumann (2), and Heisenberg (3) are time reversible. This means that a process that goes backward in time is formally admissible. Time-reversal symmetry is a characteristic feature of so called closed (or isolated) systems, i.e. systems that exchange no energy with the environment. What happens if the system is not closed? Any realistic system is never perfectly isolated. Such systems are considered to be open and Andrzej Kossakowski was awarded precisely for "developing the theory of open quantum systems".

The dynamics of open quantum systems are no longer time reversible. A typical example of a process that cannot be time reversed is spontaneous emission; when an atom relaxes from an excited state to ground state it emits. Many physicists attempted to find a coherent description of open quantum systems. A variety of approaches based on so-called Master Equations were proposed. Some of these equations provided solutions consistent with experimental data. Others were mathematically inconsistent and gave wild predictions. The main problem was a lack of a universal principle on the way to construct a mathematically sound equation which makes predictions consistent with experimental data. 
Andrzej Kossakowski began his research in this field in the late 1960s. His first important results were published in 1972 (one paper, Kossakowski 1972, was published in Reports on Mathematical Physics which was, and remains, edited in Toruń). At the beginning of 1970s Andrzej Kossakowski was invited by Professor Hermann Haken to Stuttgart University, where he met Vittorio Gorini from Milan. Gorini and Kossakowski became close collaborators and firm friends and Gorini has regularly visited Kossakowski in Toruń. In 1974 Kossakowski and Gorini were invited to Austin (Texas) by Professor George Sudarshan, who was also interested in open quantum systems at the time and was already well known for his contributions to quantum optics (Sudarshan-Glauber distributions) and elementary particles (weak interactions). In Austin Kossakowski, Gorini and Sudarshan prepared a fundamental paper "Completely positive dynamical semigroups of N-level systems" which was published in 1976 in The Journal of Mathematical Physics (Kossakowski 1976). Interestingly, this paper was published exactly 50 years after Schrödinger's paper. This provides the most general equation governing the evolution of open quantum system in the Markovian regime and clearly generalises the von Neumann equation (2).

$$
\dot{\rho}=-\frac{i}{\hbar}[H, \rho]+\frac{1}{2} \sum_{i, j=1}^{N^{2}-1} c_{i j}\left(\left[F_{i}, \rho F_{j}^{\dagger}\right]+\left[F_{i} \rho, F_{j}^{\dagger}\right]\right),
$$

The paper has three authors, but Kossakowski was key in developing the final form of the equation, which is based on his earlier papers from 1972.

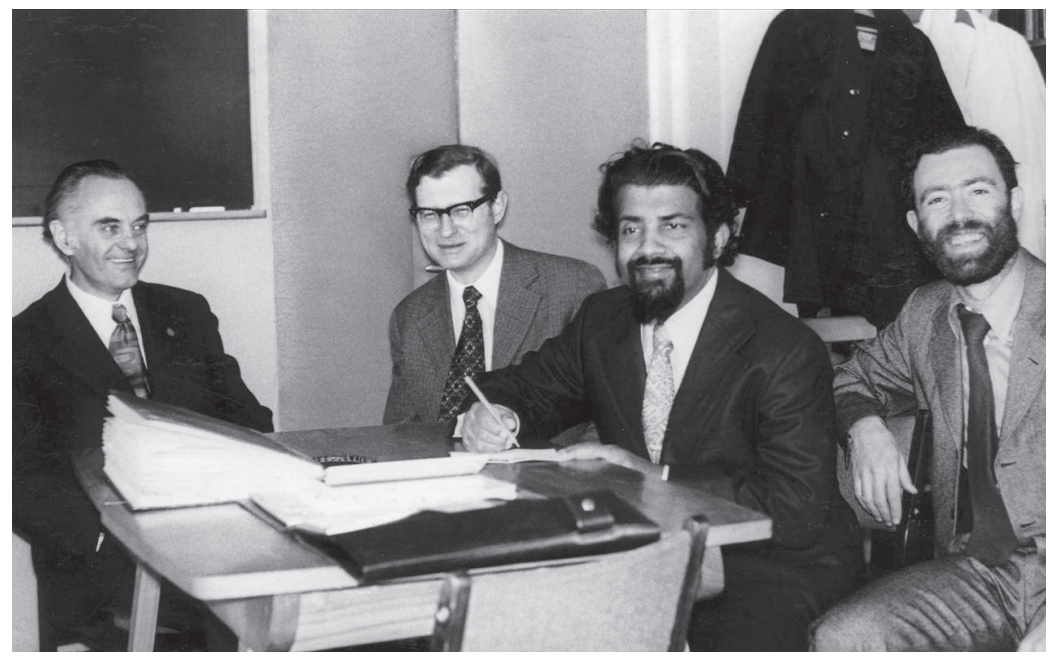

Photo 1. Picture taken in Prof. Ingarden's office (December 1975). From left to right: Roman Ingarden, Andrzej Kossakowski, George Sudarshan and Vittorio Gorini 
In the same year Göran Lindblad from Stockholm University published a paper "On the generators of quantum dynamical semigroups" in Communications in Mathematical Physics (Lindblad 1976). He derived essentially the same equation to generalise not (2) but (3). His equation reads

$$
\dot{X}=\frac{i}{\hbar}[H, X]+\sum_{i}\left(V_{i} \rho V_{i}^{\dagger}-\frac{1}{2}\left(V_{i} V_{i}^{\dagger} X+X V_{i} V_{i}^{\dagger}\right)\right) .
$$

For this reason equation (4) and (5) are both referred to as the Gorini-Kossakowski-Sudarshan-Linblad (GKSL) Master Equation.

Today the GKSL Master Equation plays an important role in Quantum Information Theory, also a Polish forte, and three FNP awards in this field went to Prof. Ryszard Horodecki (2008), Prof. Maciej Lewenstein (2011), and Prof. Marek Zukowski (2013)). Quantum Information Theory was also initiated in Torun with • Prof. Roman Ingarden's visionary paper entitled "Quantum Information Theory", which was published in Reports on Mathematical Physics again in 1976. Prof. Ingarden was for many years a head of the Department of Mathematical and Statistical Physics at Nicolaus Copernicus University. The GKSL Master Equation enables one to model important physical processes such as decoherence and dissipation. These processes play a key role in quantum communication, quantum cryptography and quantum computation. This demonstrates that Kossakowski's findings of more than 40 years ago are currently applied in modern quantum technologies.

In 2016 the Department of Mathematical Physics at Nicolaus Copernicus University organized the $48^{\text {th }}$ Symposium on Mathematical Physics entitled Gorini-Kossakowski-Lindblad-Sudarshan Master Equation - 40 Years On (cf. http://fizyka.umk.pl/smp/smp48/ for more details). The Symposium attracted more than 100 leading experts in the field of open quantum systems. Vittorio Gorini, Andrzej Kossakowski and George Sudarshan were honorary guests at the Symposium. Göran Lindbald was unable to come, but he sent us slides recalling his visit to Torun in December 1974. He participated in the Symposium on Mathematical Physics and on $5^{\text {th }}$ December and presented the basic results of his research. Andrzej Kossakowski was in Texas with Gorini and Sudarshan at the time and they were also ready with their results. On his return to Torun Kossakowski learnt of Lindblad's findings from Prof. Lech Woronowicz (FNP Prize 1993). Kossakowski and Lindblad never met. Their best opportunity to meet would have been the actual Nobel Prize ceremony in Stockholm, which Lindblad would have no excuse to miss. More information about the history of the GKLS Master Equation may be found in (Chruściński, Pascazio 2017). 


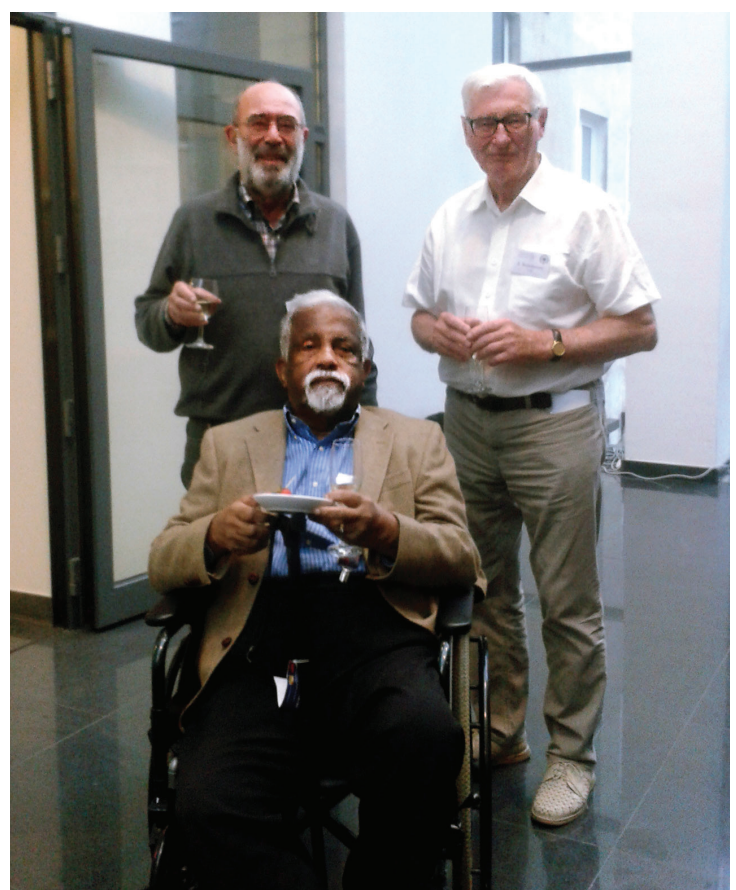

Photo 2. Picture taken during the $48^{\text {th }}$ Symposium on Mathematical Physics, Toruń, June 2016

In my opinion the GKSL Master Equation devised by Andrzej Kossakowski is one of the most important equations in $20^{\text {th }}$-Century Polish theoretical physics and he fully deserves the Foundation for Polish Science Prize.

\section{References}

Kossakowski A., 1972, “On Quantum statistical mechanics of non-Hamiltonian systems”, Reports of Mathematical Physics 3: 247.

Gorini V., Kossakowski A., Sudarshan E. C. G., 1976, “Completely positive dynamical semigroups of N-level systems", Journal of Mathematical Physics 17: 821.

Lindblad G.,1976, "On the generators of quantum dynamical semigroups", Communications in Mathematical Physics 48: 119.

Chruściński D., Pascazio S., 2017, “A Brief History of GKLS Equation”, Open Systems of Informational Dynamics 24: 1740001. 
